\title{
Calculations Relating to a Conjecture of Pólya and Schoenberg
}

\section{By Herbert S. Wilf}

In [1] it was conjectured that if the functions

$$
f(z)=\sum_{\nu=1}^{\infty} a_{\nu} z^{\nu} ; \quad g(z)=\sum_{\nu=1}^{\infty} b_{\nu} z^{\nu}
$$

are regular in $|z|<1$ and map it onto a schlicht convex domain, then the same is true of the function

$$
h(z)=\sum_{\nu=1}^{\infty} a_{\nu} b_{\nu} z^{\nu}
$$

This conjecture was tested, in the manner described below, on the ILLIAC computer of the University of Illinois. No counterexample was found, which would seem to enhance the plausibility of the conjecture, particularly in view of the fact that the test functions tended to have large, non-real coefficients, and therefore provided a reasonably stern test.

The machine program was planned and executed by Messrs. Roger A. Horn (Cornell University), Forrest R. Miller Jr. (University of Oklahoma) and Gerald Shapiro (Massachusetts Institute of Technology) who visited the Digital Computer Laboratory at Illinois during a summer program for undergraduates in Applied Mathematics sponsored by the National Science Foundation. These calculations were made possible largely by their dedication and enthusiasm.

According to the Schwarz-Christoffel mapping, the function

$$
f(z)=\int_{0}^{z} \frac{d \xi}{\left(1-e^{i \theta_{1}} \xi\right)^{\alpha_{1}} \cdots\left(1-e^{i \theta_{n}} \xi\right)^{\alpha_{n}}}
$$

$$
0 \leqq \theta_{1}, \cdots, \theta_{n}<2 \pi
$$

$$
\alpha_{j} \geqq 0
$$$$
(j=1,2, \cdots, n)
$$

$$
\alpha_{1}+\alpha_{2}+\cdots+\alpha_{n}=2
$$

maps the unit circle $1-1$ onto a convex polygon with exterior angles $\alpha_{1} \pi, \cdots, \alpha_{n} \pi$. Hence, a function $f(z)$ was constructed by (a) choosing $\alpha_{1}, \alpha_{2}, \cdots$ independently at random in $(0,1)$, stopping when $\alpha_{1}+\alpha_{2}+\cdots+\alpha_{n}>2$ (b) replacing $\alpha_{n}$ by $2-\alpha_{1}-\alpha_{2}-\cdots-\alpha_{n-1}$ (c) choosing $\theta_{1}, \theta_{2}, \cdots, \theta_{n}$ independently at random in $(0,2 \pi)(d)$ computing the first ten power series coefficients of the function (3) (a).

This process was then repeated, producing the function $g(z)$, and the first ten coefficients of $h(z)$ of $(2)$ were then calculated. In order for $h(z)$ to map $|z|<1$ onto a schlicht convex domain it is necessary and sufficient that [2]

$$
\frac{z h^{\prime \prime}(z)}{h^{\prime}(z)}+1=1+2 C_{1} z+2 C_{2} z^{2}+\cdots
$$

Received March 2, 1962. 
have positive real part in the unit circle, and for this it is necessary and sufficient that [3]

$$
\begin{gathered}
\Delta_{m}=\operatorname{det}\left(C_{i-j}\right)_{i, j=0}^{m} \geqq 0 \quad(m=0,1,2, \cdots) \\
\left(C_{-k} \equiv \bar{C}_{k}\right)
\end{gathered}
$$

Thus the $C_{j}$ in (4) were calculated recursively and the determinants $\Delta_{0}, \Delta_{1}, \cdots$, $\Delta_{9}$ were computed and tested for positivity. If the entire sequence of operations so far described be referred to as a "case," then one hundred seventy five cases were run on ILLIAC. The first one hundred ten were run precisely as described above while in the remainder the choice of the $\alpha_{i}$ was biassed toward larger values so as to explore more carefully neighborhoods of the extremal function $z(1-z)^{-1}$.

Digital Computer Laboratory

University of Illinois

Urbana, Illinois

1. G. Pólya \& I. J. Schoenberg, "Remarks on de la Vallée Poussin means and convex conformal maps on the unit circle," Pacific J. Math., v. 8, 1958, p. 295-334.

2. Z. Nehari, Conformal Mapping, McGraw Hill, 1952.

3. G. Pólya \& G. Szegö, Aufgaben und Lehrsátze aus der Analysis, Dover, 1945. 